\title{
Alternate methods for sampling in coordinate metrology
}

\author{
C E Collins, E B Fay, J A Aguirre-Cruz, and S Raman* \\ Department of Industrial Engineering, The University of Oklahoma, Norman, Oklahoma, USA \\ The manuscript was received on 10 September 2006 and was accepted after revision for publication on 23 January \\ 2007.
}

DOI:10.1243/09544054JEM734

\begin{abstract}
A variety of inspection equipment exists in industry to capture the points on a surface that will envisage manufacturing form errors. Manufacturing inspection faces a problem of finding optimal methods to capture an evenly spread distribution of points on the surface. Sampling does not yield complete information about a surface. Each material removal process leaves a unique pattern on the surface of the workpiece, which has to be taken into consideration while developing the sampling strategy. For instance, round patterns left by face milling at low feeds on flat surfaces, and spiral patterns left during surface milling and turning operations on surfaces of revolution. Two new methods have been developed to improve sampling using the coordinate measuring machine (CMM) for inspection of flat and revolved surfaces. These are the Spiral, and Hamspi sampling methods.

The Spiral method focuses on the centre of the area and uses the Archimedean spiral. Hamspi is a method that combines both the Spiral and randomized Hammersley to measure points in the middle as well as the outer zone of the workpiece. Mathematical comparisons of these methods have been made to establish feasibility. An experiment was performed to determine the accuracy of these models using two dependent variables: inspection time and minimum zone. The minimum zone was (statistically) significantly affected by only two factors: sample size and workpiece shape. The sampling time was however affected by sample size, workpiece shape, and the interaction between them. This study observed that the beginning and ending cutting zones of spherical surfaces were the most significant regions to verify. It was found that the Spiral and Hamspi methods had similar point distributions as the Hammersley method while placing more emphasis on the origin of the workpiece.
\end{abstract}

Keywords: sampling methods, minimum zone, form tolerance, sphericity, flatness forms, and spiral

\section{INTRODUCTION}

Tolerance zones must be established and verified through specifications of form, size, orientation, and location to determine whether a machined workpiece has been designed and manufactured properly [1]. Deviations occur with these processes and are caused by misalignments, machine imperfections, vibrations, temperature, and tool wear. For instance, round patterns are left by face milling at low feeds on flat

*Corresponding author: Department of Industrial Engineering, The University of Oklahoma, Norman, OK 73019, USA. email: raman@ou.edu surfaces, and spiral patterns are left during surface milling and turning operations on surfaces of revolution. Prudent sampling must be employed to measure and analyse these tolerances. Various studies have been performed in sampling such as Woo [2], Lee [3] and Rossi [4]. Sampling methods are a common way of determining errors. They are used to capture points along an object's surface. Ideally, these sampling methods are developed in accordance with the surface area, the shape of the workpiece, and tolerance specifications. The errors that occur make up the tolerance zone, which is the difference between the measured value and the fitted value. Sampling methods also need to be fast due to the cost of 
down time that occurs during sampling while still giving complete enough information about the surface in question to be useful.

Some examples of these sampling methods include the Hammersley method, random sampling, Halton-Zaremba, aligned systematic, and uniform, among others. All sampling methods are prone to error. Random sampling is useful but may not take the entire surface into account. Aligned systematic sampling is good for adapting to many shapes but encounters difficulties due to equal spacing of the points down the surface. Periodic errors, typical of manufacturing processes may not be adequately described. According to Lee [3] and Woo [2], the Hammersley method is very reliable and efficient, since simulation revealed that there was close to a quadratic reduction of the points needed to be taken by uniform sampling to have similar accuracy. The randomization of the Hammersley allows for a reduction in the risks of capturing systematic errors [3]. However, Hammersley does not place enough emphasis on the beginning and ending cutting zones as is shown in the following sections. Due to this reason, the present study focuses on applying the Spiral and the Hamspi strategies to capture the form deviations of flat and revolved surfaces.

Form measurement often involves the use of a coordinate measuring machine (CMM) to inspect the workpieces [5]. Many studies have been done utilizing the CMM. The CMM is designed to capture points along the surface. Many considerations should be made to inspect a workpiece such as the shape and size of the workpiece. The determination of the sampling method that will be employed on the part is relative to the size and shape of the workpiece being evaluated and its quality [6]. If the part needs to be inspected in more detail, the number of sampling points should increase accordingly [7]. This implies that in order to get a more accurate reading of the imperfection of the part, the sample size should increase [4].

Sample size has been demonstrated to have a direct correlation with the determined error. An infinite amount of sample points would give complete information about the surface. Having large sample sizes can incur other problems, however, such as machine drift due to heat generated by the machine. Studies have been performed to minimize sampling effort while maintaining accuracy [8]. A bound was established by Roth [9] that has been adapted to sampling methods. It illustrates that there is a maximum amount of information that can be attained from a surface. This provides for a limit in the number of useful sample points to be taken $[\mathbf{2}, \mathbf{9}]$. New methods are desired that will be efficient and reliable while overcoming the weaknesses of current methods. The ability to predict manufacturing errors occurring in a workpiece is important for sampling methods.

The form evaluation of flat surfaces has been studied by Kim [10] who illustrated a comparison of sampling methodologies in terms of measurement discrepancy and time; Badar [11-13] developed adaptive sampling methods based on optimization techniques and on the quality of the surfaces generated by end- and face-milling processes. Yang [14] applied the uniform sampling method to determine the form of error of flat surfaces. The surfaces were machined using boring, end milling, fly cutting, grinding, and shaping processes, and were analysed using the Shannon sampling method and the minimax algorithms. The Shannon sampling provided a better estimation of the form error than the minimax algorithm, since the first uses an infinite degree spline function to reconstruct the inspected surface. Round forms were studied by Rossi [4] who developed an adaptive sampling procedure to minimize the inspection time of round parts based on the information collected from a pre-sampling procedure. The screening procedure helped to detect cyclic and non-cyclic deviations such as waviness and random error that was used later on to adjust the point distribution over the surface of the inspected part.

Chetwynd [15] studied a method for offsetting a circle from the origin using limacons. The latter was adapted to spherical surfaces by Samuel [5] in which he also developed a method of determining the residuals of the sphere. Roundness measurements with a CMM were evaluated by Chan [16] to see how many points are required to truly determine circularity. Conical surfaces were studied by Prakasvudhisarn [17] and toroidal surfaces by Aguirre-Cruz [18]. Both of these studies utilized the least squares and linear optimization fitting algorithms. The least squares is a 'best fit' method of estimating the regression coefficients by reducing the sum of the squares while the minimum zone (LOPT) is a method of reducing the maximum deviation and is illustrated more in-depth by Winston [19]. Prakasvudhisarn [20] proposed a new data fitting procedure, support vector regression, which is not sensitive to the normality assumption. It was observed that the least squares is capable of overestimating the tolerance zone since it is very sensitive to outliers, while the minimum zone method is more robust since it minimizes the maximum deviations.

\section{OVERVIEW OF PRESENT WORK}

Two new methods have been developed in this paper for three-dimensional shapes. These methods are the Spiral method, and the Hamspi method. 
These will be explained in detail in the following sections. The Spiral method is a very promising new method of sampling that has some properties similar to existing systematic sequences but is an improvement over the aligned systematic sampling. Instead of lines that descend along the side of the surface, it uses the curves of the Archimedean spiral. Due to the fairly even distribution of points, it enables the sampling of the entire surface without leaving large holes in the sampling area. Depending on the parameters that one sets, the shape and tightness of the spiral are easily changeable. The advantage of using the Spiral method on a flat plate is that it could capture the pattern of the milling process. This allows for the method to focus on the machining imperfections along the surface. The advantage of using this method on spherical surfaces is that it focuses on the centre of the workpiece where much deviation could occur. There has apparently been very little research done in the area of using spirals to sample during form tolerance verification. Wieczorowski [21] has done research using spirals with a profilometer, and he found that this method greatly reduced the amount of time spent performing the experiment. No other studies over this method were found.

The Hamspi method combines both the Spiral and the randomized Hammersley methods to capture points on the surface. This method allows for various characteristics of a surface to be considered. The points on the origin of the workpiece are generated using the spiral method. Randomized Hammersley points attempt to cover the rest of the manufactured part. This permits for the centre cutting zone to be examined in detail while allowing for an even distribution of points along the rest of the surface. Such a sampling scheme could be especially useful in inspecting plates, which are clamped on the outside during machining. It has been found that surfaces tend to exhibit more minima in the middle in such cases. Hamspi is a new approach that was developed to find more specifics of what manufacturing error occurs on a surface.

Another method, called the pie method, was also studied; it utilized both the aligned systematic and random sampling to capture points along the surface of the machined part. This method has not been used in literature. Using the aligned systematic method, the surfaces were divided into equidistant areas resembling slices of a pie, such as in Prakasvudhisarn [17]. The regions contained in these areas were sampled by the random sampling method, such as in Aguirre-Cruz [18]. This was supposed to allow for more coverage of the surface than the Aligned Systematic. However, this method was not pursued further due to the lack of coverage of the surface with small sample sizes.

\section{SAMPLING METHODS}

\subsection{Spiral method}

The Spiral method follows some simple concepts. The sampling sequence follows the behavior of the Archimedean spiral, and was chosen due to its ease of manipulation and ability to reverse the direction of the spiral. The Archimedean spiral has three variables that govern the shape of the spiral: the constant $a$, which affects the radius of the spiral, the angle $\theta$, which determines the rotation of the spiral, and the constant $n$, which determines the shape of the spiral [22]. Equation 1 shows the relation between these three variables.

$$
r=a \theta^{\frac{1}{n}}
$$

The magnitude and sign of the variable $n$ affects the direction and tightness of the spiral, for example $n=1$ provides the shape of the Archimedes spiral, $n=-1$ provides the shape of a hyperbolic spiral, and $n=2$ provides the shape of a Fermat's spiral [22]. The magnitude and step size of the angle $\theta$ affect the shape of the spiral; for example, if the step size of the angle is very small, then the shape of the spiral will be perfectly round; however, if the step size of the angle is set very large, then the spiral will look similar to the Aligned Systematic strategy. It can be said that the shape of the spiral depends on the combination among these three variables, as seen in Figs 1 and 2.

Figure 1 illustrates a very simple but useful design. The constants are set with $n$ equalling 1 and $a$ equalling 0.001969 . These were set to provide simplicity and to have bounds within the actual radius of the test part, in this case, a circular plane with radius of 1.25. The shape of the spiral in Fig. 3 makes it appear very similar to the aligned systematic since it looks like five separate spiral lines, whereas in reality, it is a single spiral. An example of the aligned systematic is shown in Fig. 4. It is much more limited in scope than the spiral method. It only yields values in a line, which leaves large empty areas where much deviation could occur.
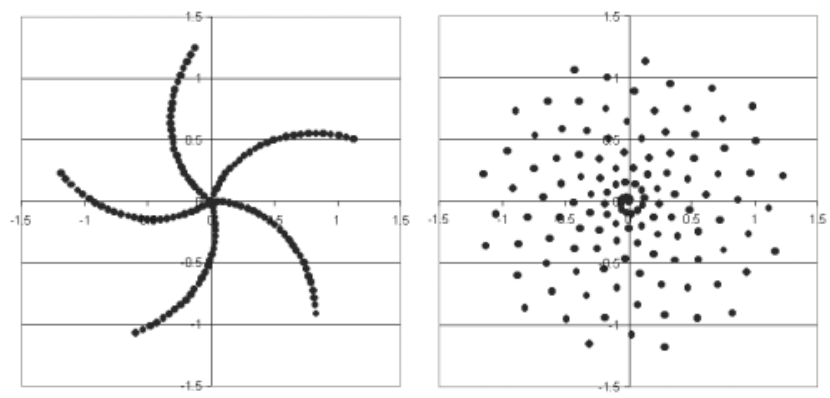

Fig. 1 Spiral with parameters $1.25 \theta$ (left), and Spiral with parameters $0.5 \theta$ (right) 

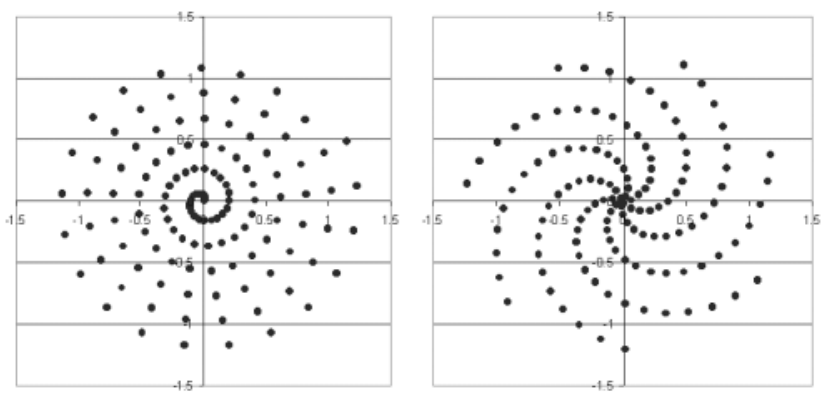

Fig. 2 Spiral with parameters .3 $\theta$ (left), and Spiral with parameters $9 \theta$ (right)

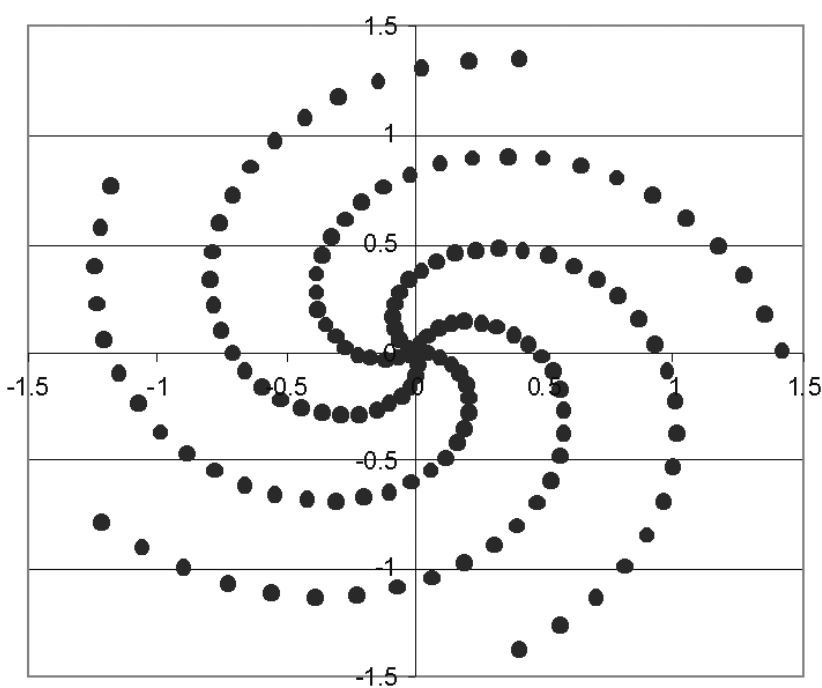

Fig. 3 The Spiral method

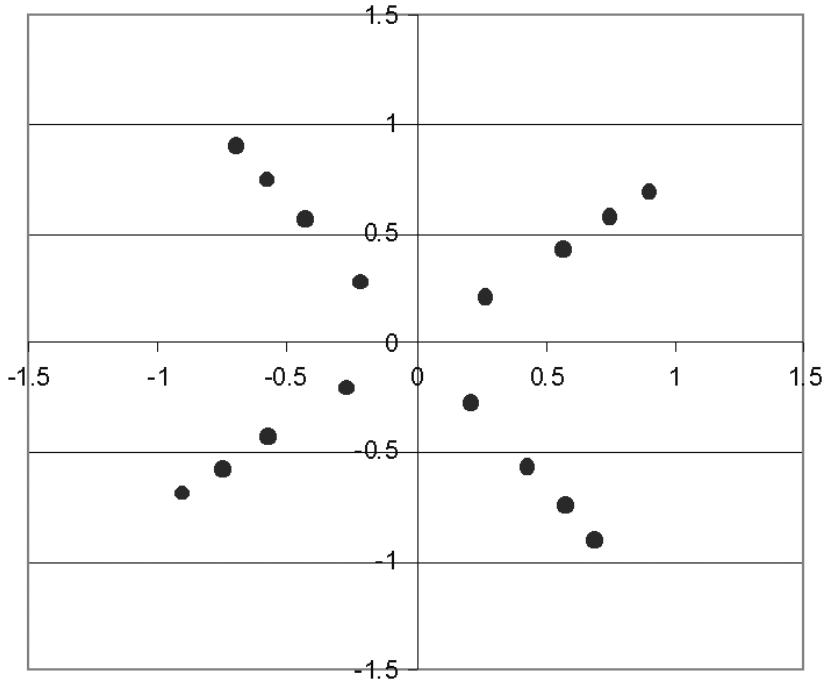

Fig. 4 Aligned Systematic method

The value for $\theta$ was set at increments of five radians. This is an arbitrary value that can very much affect the shape of the spiral. The increment can make the spiral appear similar to the Hammersley method,

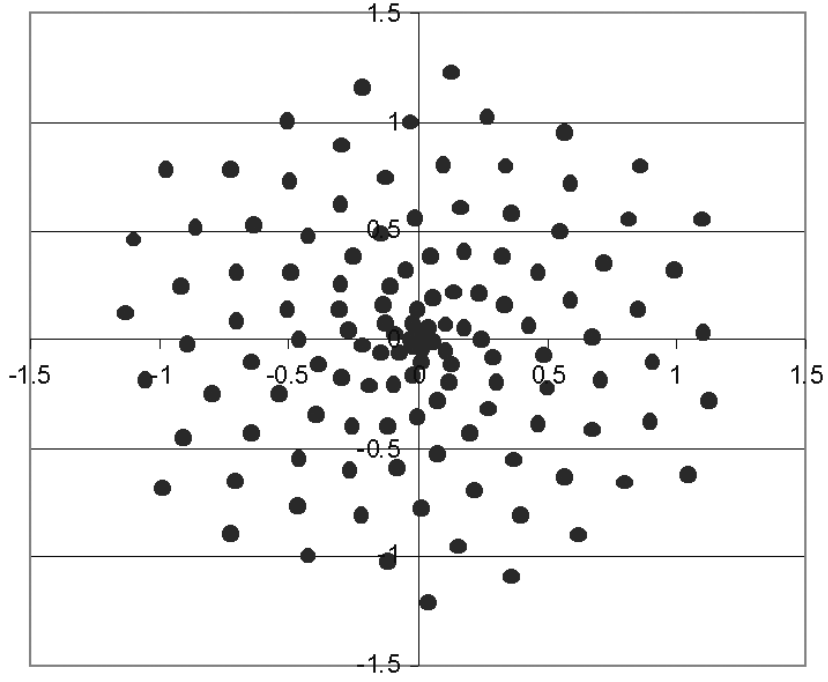

Fig. 5 Spiral parameter- $\theta$ with 1 spacing

the aligned systematic method, or a combination of the two. The value of five radians per increment was useful to make a pattern that was very similar to the aligned systematic method but that would be able to yield more useful data. Once the increment value is set and the constants are determined, the values of $r$ can be determined. Figure 5 shows the actual spiral used in the experiment. It has a more even distribution about the surface of the object, thus allowing for the measurement of the whole surface.

The shape of the spiral is easily modified depending upon what parameters are desired. For this experiment, the value for $\theta$ was equally distributed to the number of points desired to be collected. The $r$ value was set as the ideal radius of the object while the $a$ value was determined with these values. A numerical example of this is setting $r$ equal to 1.25 and $\theta$ equal to 64 , adding a random number to $\theta$ such as 0.67 , and using equation (1) with $n$ equalling 1 to yield the value 0.019 . This allowed for easy determination of scale. The following procedure explains the steps needed to convert the polar coordinates of the spiral method into Cartesian coordinates. This procedure is necessary since the coordinate measuring machine used in this research uses Cartesian coordinates.

The Spiral sampling sequence can be transformed to 2D Cartesian coordinates by using equations (2) and (3).

$$
\begin{aligned}
& x=r_{i} \cos \left(\theta_{i}\right) \\
& y=r_{i} \sin \left(\theta_{i}\right)
\end{aligned}
$$

Table 1 gives an example of ten points generated by the Spiral method with the previously stated constants being implemented, where the $\theta$ value was set to increments of 1 , the $r$ was set as the ideal 
Table 1 Ten spiral points

\begin{tabular}{|c|c|c|c|c|c|}
\hline Theta & $r$ & $x$ & $y$ & $\cos (\theta)$ & $\sin (\theta)$ \\
\hline 0 & 0.00000 & 0.00000 & 0.00000 & 1.00000 & 0.00000 \\
\hline 1 & 0.12500 & 0.06754 & 0.10518 & 0.54030 & 0.84147 \\
\hline 2 & 0.25000 & -0.10404 & 0.22732 & -0.41615 & 0.90929 \\
\hline 3 & 0.37500 & -0.37125 & 0.05292 & -0.98999 & 0.14112 \\
\hline 4 & 0.50000 & -0.32682 & -0.37840 & -0.65364 & -0.75680 \\
\hline 5 & 0.62500 & 0.17729 & -0.59933 & 0.28366 & -0.95892 \\
\hline 6 & 0.75000 & 0.72013 & -0.20956 & 0.96017 & -0.27942 \\
\hline 8 & 1.00000 & -0.14550 & 0.98936 & -0.14550 & 0.98936 \\
\hline 9 & 1.12500 & -1.02500 & 0.46363 & -0.91113 & 0.41212 \\
\hline
\end{tabular}

Table 2 Ten randomized spiral points

\begin{tabular}{|c|c|c|c|c|c|c|}
\hline Theta + rand & Theta & $r$ & $x$ & $y$ & $\cos (\theta)$ & $\sin (\theta)$ \\
\hline 0.645758 & 0 & 0.00252 & 0.00201 & 0.00152 & 1.00000 & 0.00000 \\
\hline 1.645758 & 1 & 0.00642 & -0.00048 & 0.00640 & 0.54030 & 0.84147 \\
\hline 2.645758 & 2 & 0.01032 & -0.00908 & 0.00491 & -0.41615 & 0.90929 \\
\hline 3.645758 & 3 & 0.01422 & -0.01245 & -0.00687 & -0.98999 & 0.14112 \\
\hline 4.645758 & 4 & 0.01813 & -0.00121 & -0.01808 & -0.65364 & -0.75680 \\
\hline 5.645758 & 5 & 0.02203 & 0.01770 & -0.01311 & 0.28366 & -0.95892 \\
\hline 6.645758 & 6 & 0.02593 & 0.02424 & 0.00919 & 0.96017 & -0.27942 \\
\hline 8.645758 & 8 & 0.03373 & -0.02400 & 0.02369 & -0.14550 & 0.98935 \\
\hline 9.645758 & 9 & 0.03763 & -0.03672 & -0.00825 & -0.91113 & 0.41211 \\
\hline
\end{tabular}

radius of the object, and the $a$ was found by solving equation (4).

$$
a=\frac{r}{\theta^{1 / n}}
$$

From equation (1), $n$ was set at 1 , the $a$ was set at 0.125 , and the $\theta$ was set as 10 , which is the number of the points being measured for the following tables. This yielded the $r$ value, which was used to compute the $x$ and $y$ values.

Similar to the manner in which Lee [3] randomized the Hammersley sequence, the Spiral sequence was randomized. This allows for multiple samples to be taken without always using the same points. The origin $(0,0)$ should be maintained throughout the samples since the origin of the part is very important to the study of its surface. To allow for this, equation (5) is used to randomize the value for $a$. The $r$ value is held constant as the ideal radius of the object, the $\theta$ value is set as the total number of points being taken, and a random number between zero and one is added to the $\theta$ value. This gives the equation

$$
a=\frac{r}{(\theta+\text { rand })}
$$

Table 2 shows the Spiral sequence with randomized points with the $a$ value being randomized with the addition of 0.645758 and using as $a$ value of 0.003178 .

The Spiral method is very useful. By taking samples that spiral around an object, the object's integrity and conformation to the desired shape can be determined. In turning processes, many problems can arise, such as machine misalignment, tool wear, and machine imperfection. By adapting this strategy to the surfaces in question, it may be possible to help predict which errors the machine might be making. By combining this sampling strategy with the minimum zone fitting algorithm, much useful data can be interpreted.

\subsection{Hammersley method}

The Hammersley method is a well used sampling method. It is viewed as one of the better methods of sampling surfaces since it allows for a nearly quadratic reduction of points while presenting similar accuracy when compared to the uniform method $[2,3]$. It is useful since it has a fairly even distribution, and yet could be limited while measuring in the centre of the object. This leads to a loss in reliability since this is a critical area for surface determination. The origin is often where cuts either begin or end. Figure 6 illustrates how this centre of the object can be missed.

The Hammersley method uses the following equations for determining points

$$
\begin{aligned}
& x_{i}=s_{i}=\frac{i}{N} \\
& y_{i}=t_{i}=\sum_{j=0}^{k-1} b_{i j} 2^{-j-1}
\end{aligned}
$$




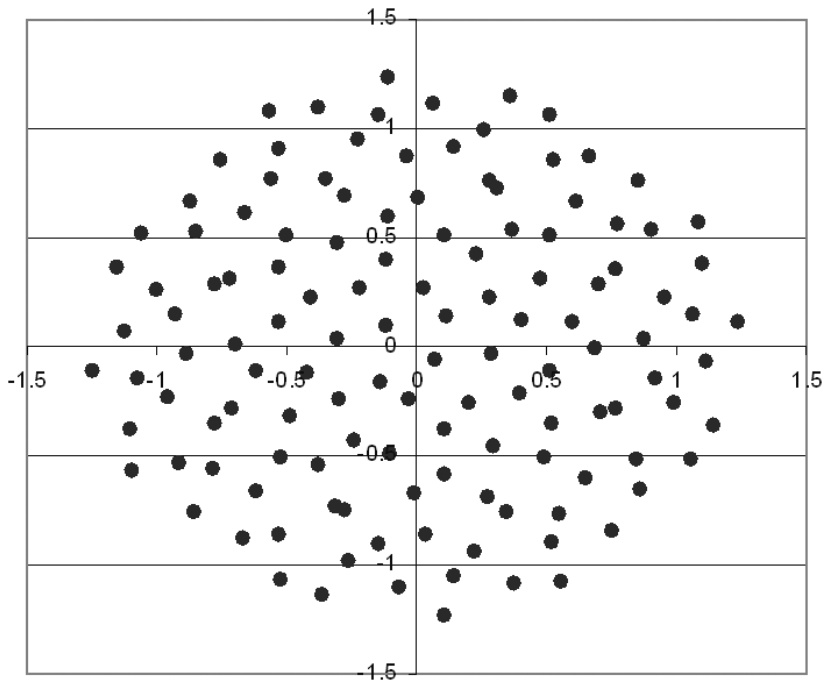

Fig. 6 Hammersley method

where $N$ stands for the total desired number of points, $i$ determines the $i$ th point that is found on a range between 0 and $N-1$, and $b_{j}$ is the binary position where $j$ goes from 0 to $k$. The $k$ value is the total number of bits and is found by equation (8).

$$
k=\left[\log _{2} N\right]
$$

For easy computing, a chart is made to list these values. To randomize the points, a random number is generated in MicroSoft Excel ${ }^{\mathrm{TM}}$ between 0 and 1 and is added to the numbers for the points. All of the points will have the same random number added.

There are four main steps in generating points for round surfaces.

Step 1 Create the points with equations (6) and (7). Step 2 Randomize the points.

Step 3 Convert the points into polar coordinates using the following equations

$$
r_{i}=\sqrt{y_{i}} R
$$

where $\mathrm{R}$ is the ideal radius of the sphere.

$$
\theta_{i}=2 \pi x_{i}
$$

Step 4 Convert these points back to Cartesian coordinates using equations (2) and (3).

This procedure is necessary due to the way that the CMM interprets input information.

\subsection{Hamspi method}

A machinist must always check for errors in the machine that could affect the making of a workpiece. The Hamspi sampling method was developed with this consideration. To study tool wear, vibration, misalignment, and machine imperfection, the start and the end of the workpiece should be analysed when performing the sampling studies. Utilizing both

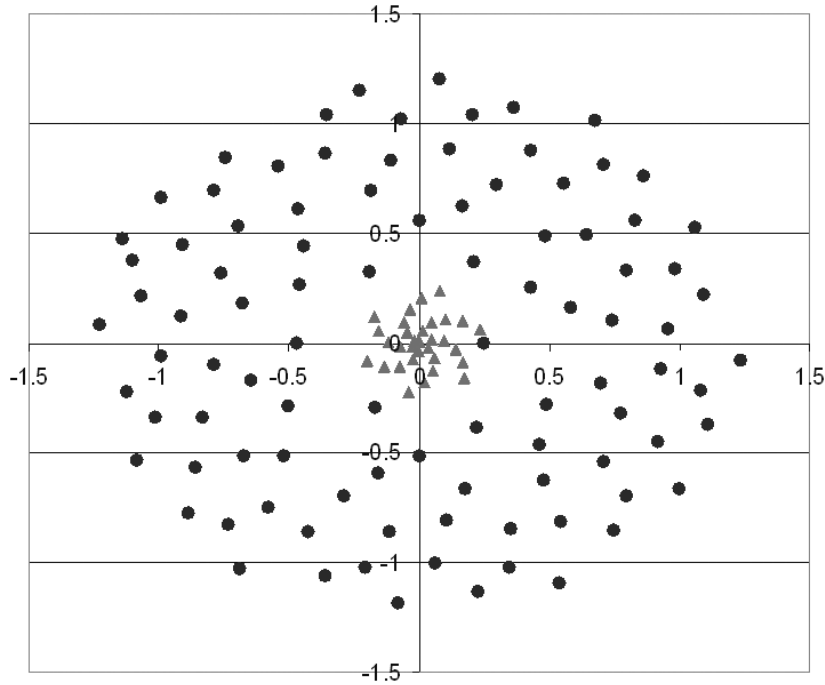

Fig. 7 Hamspi method showing the Hammersley approach

the Hammersley and Spiral methods, the Hamspi method will take these areas into consideration.

The surface and centre of round workpieces should be considered when verifying the tolerance zones. The Hammersley method has proven to be reliable in its ability to verify a surface, $[6,12,17,18]$. However, it does not place enough emphasis on the centre of the workpiece. The Hammersley method is utilized on the outer areas of the workpiece for its broad and even distribution of points along the surface. To intensify the sampling towards the centre of the workpiece, the spiral method is incorporated at the centre. The focus of this sampling strategy is to better obtain the errors found at the centre of the workpiece, where the tool touches the manufactured part either first or last. A diagram of the Hamspi method is shown in Fig. 7.

The spiral points are found by using equations (1) and (2). The areas covered by the Spiral method were set at the centre of the surface. The Hammersley two-dimensional measuring points are found by the same equations as the normal Hammersley method, except that equation (9) is changed with the following adaptation to make room for the spiral points and to avoid overlapping.

$$
r_{i}=\sqrt{y_{i}} R+C
$$

Here $C$ is the radius taken up by the Spiral method. The spiral follows equation (1) with the desired radius set as $C$. The measuring points for each part are found by the same equations as for that particular method.

In this research, one quarter of the total points were set as spiral points while the remaining were set for the Hammersley sequence. This distribution is reasonable for practice. It is expected to place emphasis on the centre of the object while still 
having a good distribution over the rest of the surface. This should yield more information than using Hammersley alone.

\section{JUSTIFICATION OF SAMPLING METHODS}

For justifying the use of these methods, it is necessary to show the distribution of points in a way that will illustrate the dependability of sampling the surface. Woo [2] used a method of determining the residual of the Hammersley method to prove that it presented a good distribution of points that would be reliable. The proposed error followed equation (12).

$$
\text { residual }=t_{1} * t_{2}-\sum_{i=1}^{N} x\left(P_{i}\right)
$$
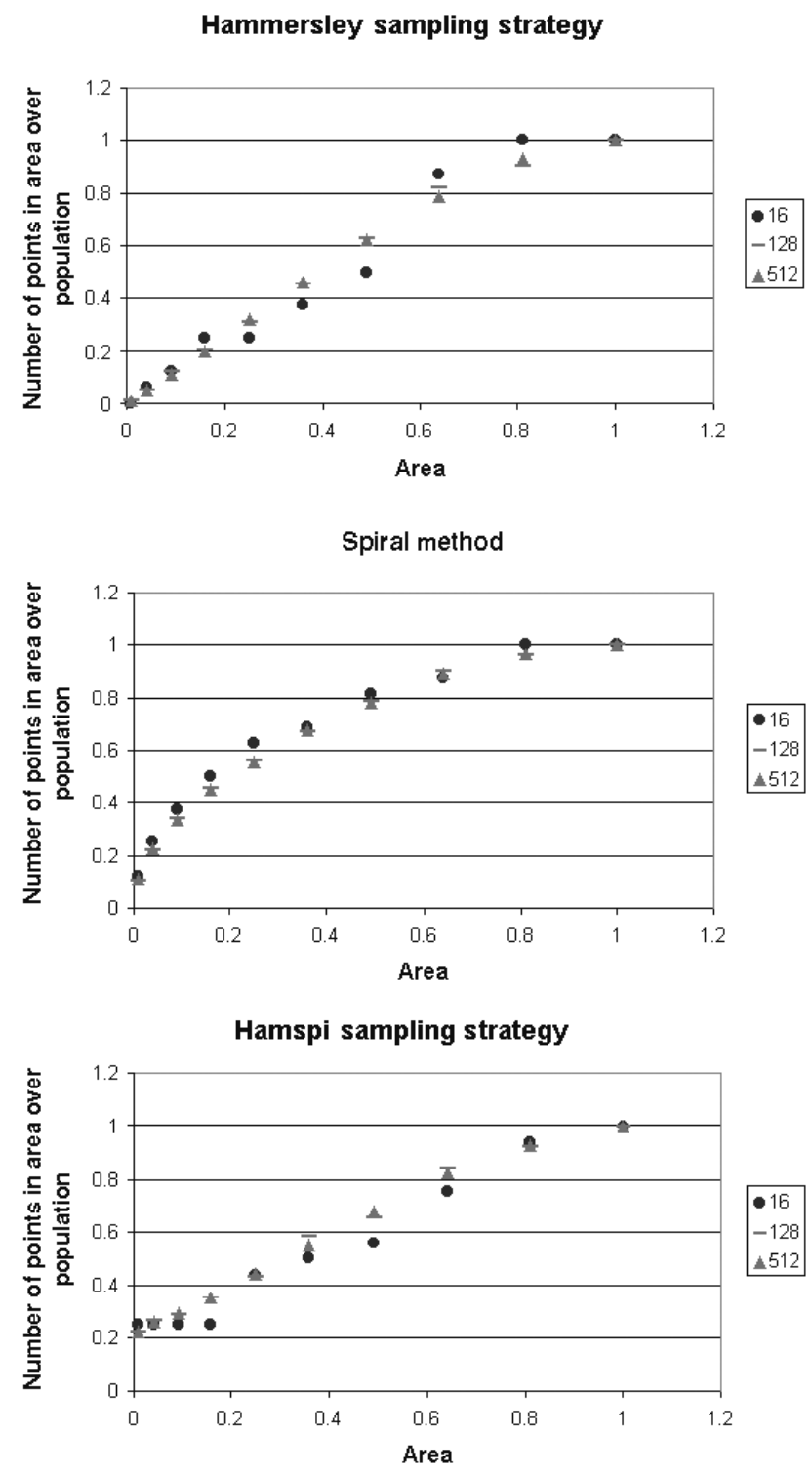

Fig. 8 Distribution of points per area
The above equation (12) illustrates the difference between the inspected area and the number of points enclosed in that region. The values of $t$ are scaled to be from 0 to 1 . Each value of $t$ corresponds with a square of sides of distance $t$. These squares enclose points that are within that given region and thus are used to determine the point distribution.

The origin is set to the centre of this square. Figure 8 illustrates the differences in point distribution between the Hammersley, Spiral, and Hamspi methods. The Hammersley method has a good distribution but lacks some emphasis on the 20 per cent closest to the origin. The Hamspi method shows a similar pattern to the Hammersley method while placing more emphasis on the 20 per cent closest to the origin. The Spiral method shows a slight curve giving a fairly even distribution of points. Both the Spiral and Hamspi place more emphasis on the 20 per cent area closest to the origin. Path planning and fitting for flatness have been investigated significantly in the literature and are not repeated here. The spherical form measurement is detailed in the next section. However, it must be noted that the sampling strategy is the subject of this paper.

\section{SPHERICAL FEATURE MEASUREMENT}

Spherical surfaces are used frequently for many part features in industry. Industrial applications include parts in engines, bearings, and Van de Graaf generators. While inspecting spheres for tolerance error, usually the radius, origin, roughness, and sphericity are evaluated. Forging, turning, form turning, milling, grinding, and lapping can each be employed to make spherical features with varying levels of finish and tolerances.

The CMM employed in this research has the capability of inspecting an object in an automatic mode. The automatic inspection requires the user to input three types of coordinates: the $\mathrm{z}$ plane, the positioning, and the measuring coordinates, which are used by the machine to follow a collision-free path. The $\mathrm{z}$ plane coordinates are located at the same height and are located exactly above the positioning coordinates, which means that they share the same $x$ and $y$ values. The height of the $\mathrm{z}$ plane is determined according to the highest point of the inspected part, for example, if the measuring part is a sphere with radius of $30 \mathrm{~mm}$, then the location of the $\mathrm{z}$ plane will be at $40 \mathrm{~mm}$, which is $10 \mathrm{~mm}$ above the inspected sphere. The distance between the sphere and the $\mathrm{z}$ plane is used by the CMM to position the stylus probe above the positioning coordinates. The positioning coordinates are obtained from the values of the measuring coordinates and follow the same shape as the object being inspected. Once the CMM 
locates the positioning point on the $\mathrm{z}$ plane, the machine lowers the stylus probe until it reaches the positioning coordinate. Then, the machine moves the probe closer to the inspected object until it reaches the measuring point. The approaching vector from the positioning point to the measuring point is normal to the surface. Thus, the measuring and positioning coordinates are located over the normal vector of the surface.

The $x y z$ components of the measuring coordinates are determined separately. First, the $x$ and $y$ values are obtained from the sampling method, while the $z$ values are determined based upon the shape of the object in question. For a sphere, the $z_{i}$ measuring values are found following equation (13)

$$
z_{i}=\sqrt{R^{2}-\left(x_{i}^{2}+y_{i}^{2}\right)}
$$

where $R$ is the ideal radius of the sphere and $x_{i}, y_{i}$ are obtained from the sampling method.

As mentioned before, the positioning coordinates are also an important part to the sampling strategy, since they create a safety zone for the probe. The steps needed to find the positioning coordinates are as follows.

Step 1 Calculate the angle $\gamma_{i}$ that determines the position of the normal vector of the $i$ th measuring point, which is found using equation (14)

$$
\gamma_{i}=\tan ^{-1}\left(\frac{z_{i}}{\sqrt{x_{i}^{2}+y_{i}^{2}}}\right)
$$

where $x_{i}, y_{i}$, and $z_{i}$ are the value of the measuring coordinates obtained above.

Step 2 Calculate the horizontal $x y_{p}$ and the vertical $z_{p}$ components of the positioning coordinates, which are obtained from equations $(15,16)$.

$$
\begin{aligned}
& x y_{p}=(R+A) \cos (\gamma) \\
& z_{p}=(R+A) \sin (\gamma)
\end{aligned}
$$

where $R$ is the ideal radius of the sphere and $A$ is the offset distance between the measuring and positioning coordinates.

Step 3 Break up the horizontal components into $x_{p}$ and $y_{p}$, as shown in equations $(17,18)$.

$$
\begin{aligned}
& x_{p}=x y_{p} \cos (\theta) \\
& y_{p}=x y_{p} \sin (\theta) .
\end{aligned}
$$

Step 4 Calculate the $\mathrm{z}$ plane coordinates, which allow for a zone where the CMM can move quickly between that plane and the positioning coordinates. The $\mathrm{z}$ plane greatly reduces the time required for the sampling while still keeping a safety zone so that the machine will not break the tip of the stylus. The horizontal components of the $\mathrm{z}$ plane coordinates

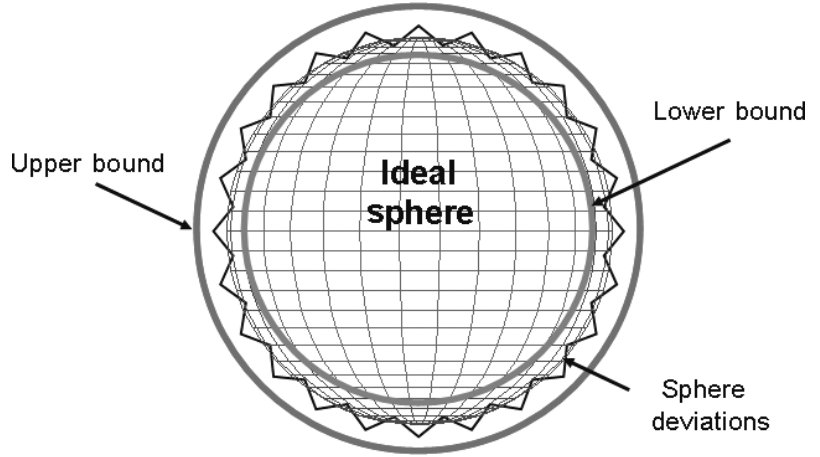

Fig. 9 Minimum zones of a sphere

are the same as the positioning points, while the vertical component depends on the highest point of the inspected part, as mentioned before.

The measuring coordinates obtained from the CMM are analysed using a linear sphericity model obtained from Samuel [5]. The model obtains the deviation by comparing the Euclidian distance $r_{i}$ of the $i$ th measuring point against an ideal value $R$ of the radius of the sphere and the origin offsets $x_{0}$, $y_{0}$ and $z_{0}$. Equation (19) shows the model used to calculate the form deviations of the sphere.

$$
d_{i}=r_{i}-\left[R+x_{0} \cos \theta \cos \alpha+y_{0} \sin \theta \cos \alpha+z_{0} \sin \alpha\right]
$$

Finally, the tolerance zone $h$ is calculated as the maximum deviation minus the minimum deviation, as seen in equation (20). The tolerance zone for the sphere is illustrated in Fig. 9.

$$
h=\left(d_{i} \max -d_{i} \min \right)
$$

\section{FLAT FEATURE MEASUREMENT}

The process needed to verify the form tolerance of the flat surface does not differ too much from the procedure to verify spheres. The three types of coordinates, z-plane, positioning, and measuring coordinates, are still needed. The main difference is the location of the positioning coordinates, which uses the form of a plane instead of a sphere. Therefore, the $\mathrm{x}$ and $\mathrm{y}$ components of the measuring, positioning and $\mathrm{z}$ plane coordinates will be the same. The $\mathrm{z}$ component will change according to the buffer zone required by the CMM.

The measuring coordinates obtained from the flat surface are analysed using the linear flatness model obtained from Badar [11], as seen in equation (21). Finally, the tolerance zone of a flat surface is calculated following equation (20), which is the same way the sphericity error was calculated.

$$
d_{i}=\left[z_{i}-\left[l x_{i}+m y_{i}+c\right]\right] / \sqrt{l^{2}+m^{2}+1}
$$




\section{EXPERIMENTAL DESIGN}

The two forms that were used in the experimental design to evaluate the sampling strategies were the sphere and the plate. The workpieces used for this experiment were randomly selected among a selection of identical workpieces. A Brown and Sharpe MicroVal $\mathrm{PFx}^{\mathrm{TM}} 454$ coordinate measuring machine was used for this experiment, which holds a sapphiretipped stylus with a diameter of $0.7 \mathrm{~mm}$ (M2 A-50030577). The accuracy and repeatability of the machine is within $0.00381 \mathrm{~mm}$. The same fixtures were used to hold both the sphere and the plate. The factors surrounding the CMM at all times included the room temperature, fixtures, tightness of the workpiece, and the point utilized for the 'Home' function. The software used by the CMM to measure the points was TUTOR ${ }^{\mathrm{TM}}$ for Windows ${ }^{\circledR}$ 98. The probe qualification consisted of determining the stylus radius. Before initializing the experiment, the reference offset was established to maintain the same parameters for all measurements. In addition, the origin of the machine was translated to the geometrical origin of the inspected part, in this case, to the centre of the sphere and to the centre of the flat plate. This procedure was necessary due to the fact that the Spiral and Hamspi strategies generate the measured points with respect to the origin of the part. However, it can be said that the points obtained from the sampling strategies can be modified in order to comply with the geometrical origin of the inspected parts.

Independent variables consisted of three sample sizes, three sampling strategies, and two fitting algorithms. The sample sizes were 16, 128, and 512. The three sampling strategies evaluated were randomized Hammersley, spiral, and the Hamspi method. The algorithms used for fitting were the least squares and the linear optimization, which were used only in the analysis of the dependent variable called 'minimum zone', defined as the difference between the measured value and the fitted value. The second dependent variable was the time of inspection that occurs from the time the machine starts to operate to when it stops moving.

The point coordinates collected from all factor combinations by the CMM were prepared for analysis using Microsoft Excel ${ }^{\mathrm{TM}}$. Then, the data were imported into Matlab ${ }^{\mathrm{TM}}$ that was used to calculate the zone of error of the sphere and flat plates. The calculated tolerance zones were analysed using the statistical procedure analysis of variance (ANOVA). The evaluation was performed using SAS ${ }^{\mathrm{TM}}$ for Windows ${ }^{\circledR}$. The level of significance for the statistical evaluation in ANOVA was set to 0.05. A review of the data was needed in order to determine the significant interactions for this experiment.

\section{RESULTS AND ANALYSIS}

The independent factors were analysed to determine their effect on the dependent variables through ANOVA. For the dependent factor of the time of inspection, it was found that the sample size, the workpiece shape, and the interaction between these two factors were significant. It can be seen that the sampling strategies were not significant; however, this means that the two proposed sampling strategies, spiral and Hamspi methods, are as time-efficient as the well known Hammersley method. These are illustrated below in Figs 10-12.

The analysis of the dependent variable minimum zone was performed twice since it was found that the hemispherical specimens used in this study were not manufactured according to specifications. The specimens should have been machined with radii of $31.75 \mathrm{~mm}$ (1.25 inches); however, it was found that the specimens had a radial height of $28.575 \mathrm{~mm}$ (1.125 inches), which was the cause of outliers in the data collected on the edges of the hemisphere. For this reason, the spherical specimen was verified using a more accurate inspection equipment, called the Accordion Fringe Interferometer, which uses the projection of laser fringes with

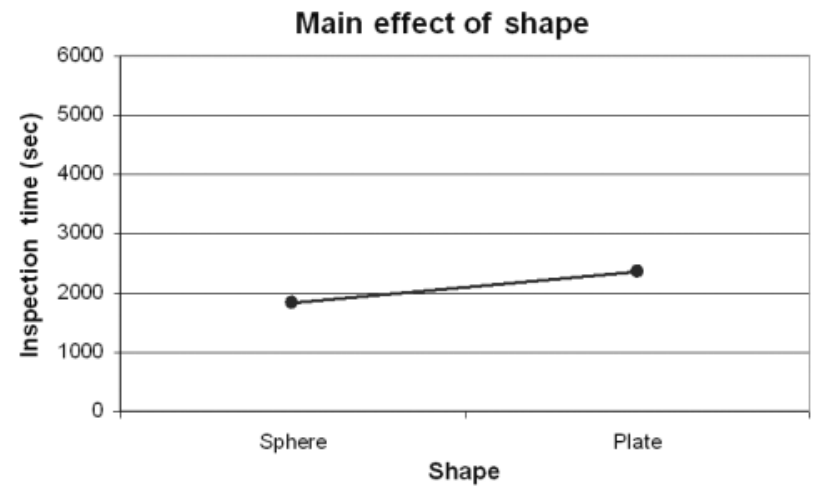

Fig. 10 Main effect of sample size on time

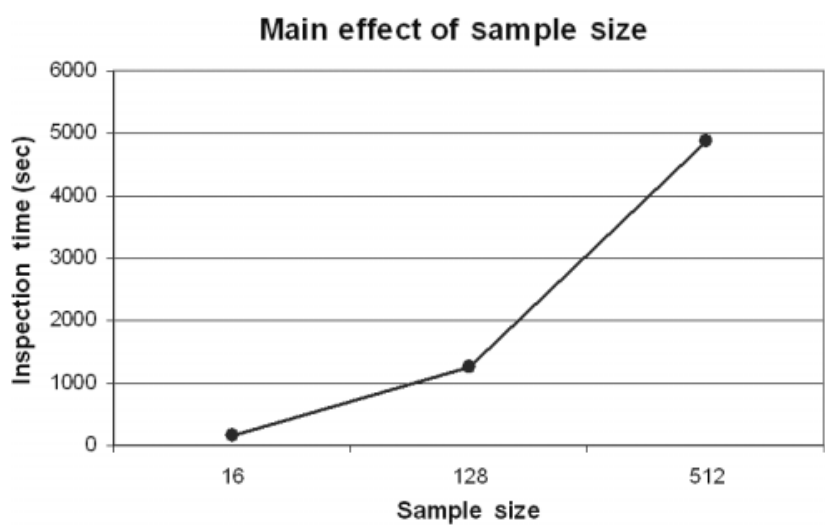

Fig. 11 Main effect plot of sample size on time 


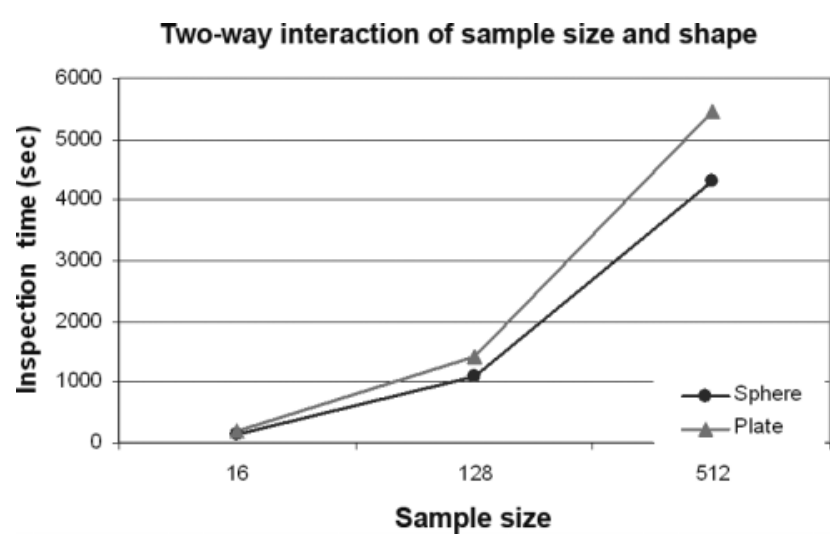

Fig. 12 The two-way interaction of sample size and shape on time

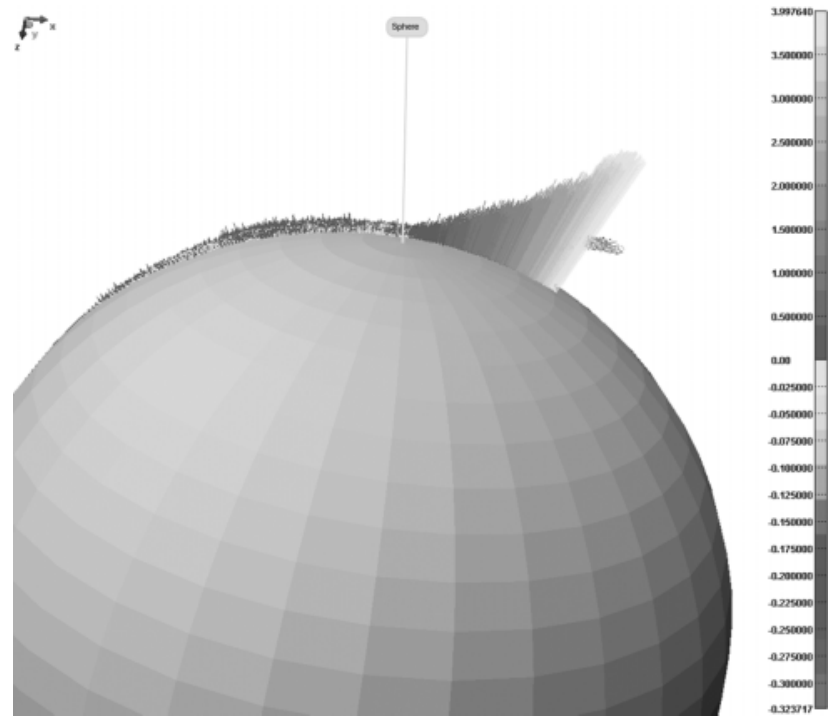

Fig. 13 Form errors of hemispherical specimen

different thicknesses to capture the inspected part. Figure 13 shows the form deviations on the edge of the hemisphere that was verified using the laser interferometer.

The first analysis showed significant differences for the factor sample size, shown in Fig. 14. The second analysis was performed by removing the outliers from the data, which were found only in sample sizes of 128 and 512. A total of six and eight outliers were removed from sample size 128 and 512, respectively. The results obtained from the experiment showed significant differences in factors sample size and fitting algorithm: the sample size showed the same effect as the first analysis, while the fitting algorithm showed that the minimum zone outperformed the least squares method.

The residuals were plotted with respect to the $x$ and $y$ Cartesian coordinates taken on the surface, which provided important information regarding

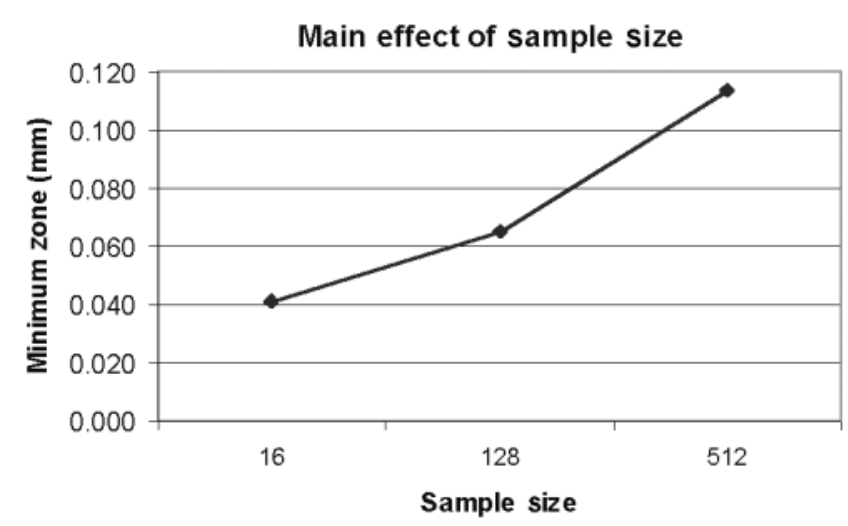

Fig. 14 The main effect of sample size on $M Z$

each shape. The plate was found to have a saddle shape and the sphere was found to have significant regions of over- and under-machining in the beginning and ending cutting zones. This in itself provides justification for non-uniform sampling. Table 3 gives the results for the minimum zone values and Table 4 summarizes the results for the inspection times.

\section{CONCLUSIONS AND RECOMMENDATIONS}

With regards to the inspection time, the statistical analysis using SAS ${ }^{\mathrm{TM}}$ showed significant differences for the main factors of shape and sample size and the interaction between them. As was expected, the sample size of sixteen was the fastest. For all sample sizes, the sphere was slightly faster than the plate. With regards to the minimum zone, the only statistically significant factor determined by SAS ${ }^{\mathrm{TM}}$ was sample size. The sample size of 512 typically produced the largest minimum zones, since it was collecting more information from the surface of the part. The sphere showed a higher zone of error than the plate. This confirms that the sphere is more complex to manufacture than the plate and therefore prone to more errors. The output revealed that the sampling method is not a key component in determining the better minimum error estimation among these methods. The large outliers that occurred are the reason that the SAS program did not detect any significant differences between the linear optimization and the least squares fitting algorithms.

The spiral and Hamspi methods are very promising new techniques for sampling surfaces. They are powerful procedures that will be able to provide more useful information about a surface while optimizing the efficiency. They present similar point distribution to the well known Hammersley method, while emphasizing the centre of the workpiece, and hence, show much promise for development and application. 
Table 3 Main experiment minimum zone values

Sphericity values (mm)

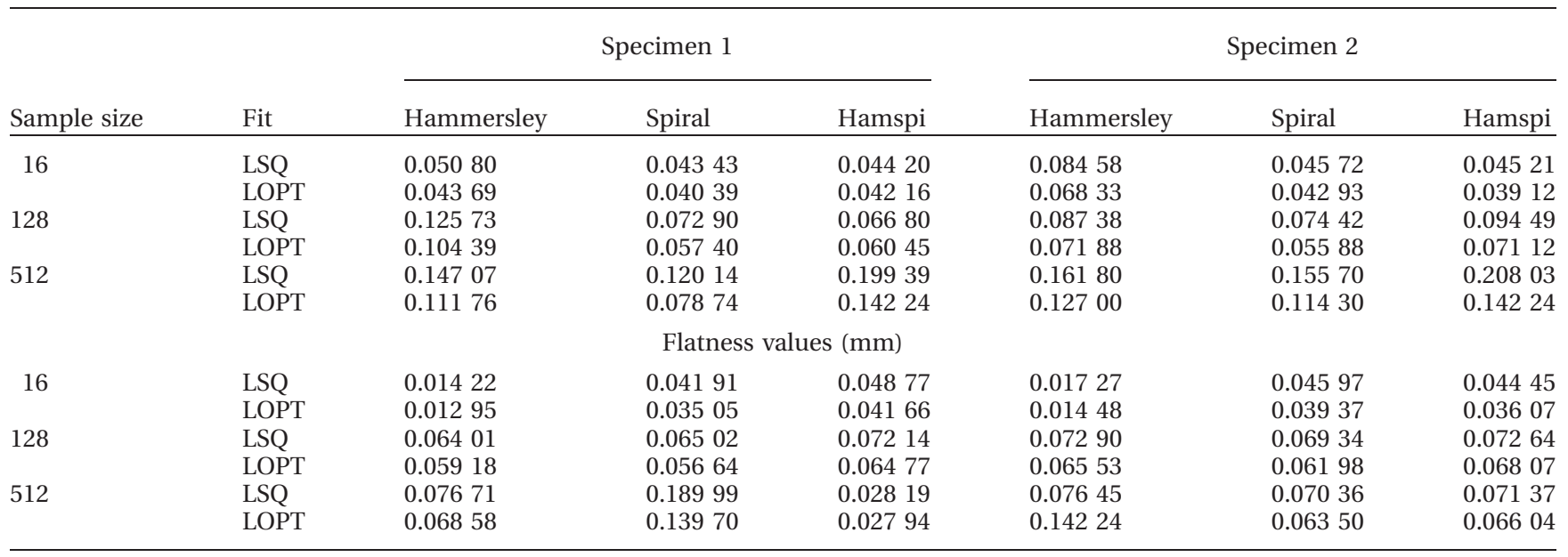

Table 4 Main experiment inspection time

Inspection time for sphere form (sec)

\begin{tabular}{|c|c|c|c|c|c|c|}
\hline & \multicolumn{3}{|c|}{ Specimen 1} & \multicolumn{3}{|c|}{ Specimen 2} \\
\hline & Hammersley & Spiral & Hamspi & Hammersley & Spiral & Hamspi \\
\hline 16 & 151 & 148 & 148 & 147 & 145 & 147 \\
\hline 128 & 1107 & 1080 & 1070 & 1098 & 1080 & 1063 \\
\hline 512 & 4336 & 4296 & 4271 & 4336 & 4313 & 4238 \\
\hline \multicolumn{7}{|c|}{ Inspection time for flat form (sec) } \\
\hline 16 & 181 & 186 & 187 & 183 & 185 & 182 \\
\hline 128 & 1363 & 1382 & 1634 & 1359 & 1375 & 1345 \\
\hline 512 & 5475 & 5730 & 5283 & 5429 & 5460 & 5339 \\
\hline
\end{tabular}

For future studies there are many areas and factors to inspect. There is much use to determining whether or not a global pattern exists in the characteristics of beginning and ending cutting zones in spherical surfaces. This would mean that by emphasizing these two regions, sample sizes could be reduced saving time. The Hamspi method could be very useful in this by increasing the distance between the Spiral and the Hammersley parts. This would place more emphasis on each zone. The intensification and diversification must however be applied after suitable experience with the process used to produce the surface. Experimentation with other forms will enhance the application domain.

\section{ACKNOWLEDGEMENT}

The authors wish to acknowledge NSF under grant DMI0427966 for providing financial support for this work. The opinions expressed herein are those of the authors and not necessarily those of the NSF.

\section{REFERENCES}

1 Henzold, G. Handbook of geometrical tolerancing: design, manufacturing, and inspection, 1995 (Wiley, Chichester, New York).

2 Woo, T. C. and Liang, R. Dimensional measurement of surfaces and their sampling. Computer Aided Design, 1993, 25(4), pp. 233-239.

3 Lee, G., Mou, J., and Shen, Y. Sampling strategy design for dimensional measurement of geometric features using coordinate measuring machine. Int. J. Mach. Tools and Mf., 1997, 37(7), 917-934.

4 Rossi, A. A form of deviation-based method for coordinate measuring machine sampling optimization in an assessment of roundness. Proc. Instn Mech. Engrs, Part B: J. Engineering Manufacture, 2001, 215(B11), 1501-1518.

5 Samuel, G. L. and Shunmugam, M. S. Evaluation of circularity and sphericity from coordinate measurement data. J. Mater. Processing Technol., 2003, 139, 90-95.

6 Edgeworth, R. and Wihelm, R. G. Adaptive sampling for coordinate technology. Precision Engng, 1999, 23, 144-154.

7 Shunmugam, M. S. Comparison of linear and normal deviations of forms of engineering surfaces. Precision Engng, 1987, 9(2), 96-102. 
8 Namboothiri, V. N. and Shunmugam, M. S. On determination of sample size in form error evaluation using coordinate technology. Int. J. Prod. Res., 1999, 37(4), 793-804.

9 Roth, K. F. On irregularities of distribution. Mathematika, A: J. Pure and Applied Math., 1954, 2(1), 73-79.

10 Kim, W. S. and Raman, S. On the selection of flatness measurement points in coordinate measuring machine inspection. Int. J. Mach. Tools and Mf., 2000, 40(3), 427-443.

11 Badar, M. A., Raman, S., and Pulat, P. S. Intelligent search-based selection of sample points for straightness and flatness estimation. Trans. ASME, J. Mfg Sci. Engng, 2003, 125(2), 263-271.

12 Badar, M. A., Raman, S., and Pulat, P. S. Experimental verification of manufacturing error pattern and its utilization in form tolerance sampling. Int. J. Mach. Tools and $M f ., 2005,45(1), 63-73$.

13 Badar, M. A., Raman, S., Pulat, P. S., and Shehab, R. L. Experimental analysis of search-based selection of sample points for straightness and flatness estimation. Trans. ASME, J. Mfg Sci. Engng, 2005, 127(1), 96-103.

14 Yang, T. H. and Jackman, J. A Shannon sampling approach to form error estimation. J. Engng Mf., 2002, 216(Part B), 225-233.

15 Chetwynd, D. G. Roundness measurement using limacons. Precision Engng, 1979, 137-141.

16 Chan, F. M. M., King, T. G., and Stout, K. J. The influence of sampling strategy on a circular feature in coordinate measurements. Measurements, 1996, 19(2), 73-81.

17 Prakasvudhisarn, C. and Raman, S. Framework for cone feature measurement using coordinate measuring machines. Trans. ASME, J. Mfg Sci. Engng, 2004, 126(1), 169-177.

18 Aguirre-Cruz, J. A. and Raman, S. Torus inspection using coordinate sampling. Trans. ASME, J. Mfg Sci. Engng, 2005, 127(1), 84-95.

19 Winston, W. L. Operation research applications and algorithms, 2004 (Duxbury Press, Belmont).

20 Prakasvudhisarn, C., Trafalis, T. B., and Raman, S. Support vector regression for determination of minimum zone. Trans. ASME, J. Mfg Sci. Engng, 2003, 125(4), 736-739.

21 Wieczorowski, M. Spiral sampling as a fast way of data acquisition in surface topography. Int. J. Mach. Tools and Mf., 2001, 41, 2017-2022.

22 Weisstein, E. W. CRC Concise encyclopedia of mathematics, 1999 (Chapman \& Hall, Boca Raton).

\section{APPENDIX}

\section{Notation}

$\theta_{i} \quad$ angle that describes the position of the $i$ th point on the $x y$ plane $\theta[0,2 \pi]$

$\gamma_{i} \quad$ angle that describes the position of the $i$ th point with respect to the $\mathrm{z}$ axis $\alpha[0, \pi]$

A offset distance between the measuring and the positioning coordinates

a constant that defines the shape of the spiral

$b_{i j} \quad$ binary position of the $i$ th point and $j$ th bit

$C \quad$ the radius taken up by the Spiral method

$d_{i} \quad$ form deviation of the $i$ th point

$h \quad$ width of the tolerance zone

$k$ total number of bits needed to generate $N$ points

$l \quad$ slope of the axis of assessment between $x z$ axes

$m \quad$ slope of the axis of assessment between $y z$ axes

$N \quad$ total number of points

$n \quad$ constant that determines the direction of the spiral

$R \quad$ ideal radius of the sphere

$r_{i} \quad$ radius of the $i$ th measuring point

$r_{i} \quad$ Euclidian distance of the $i$ th measuring point

$s_{i} \quad$ horizontal component obtained from Hammersley strategy

$t_{1} \quad$ value that determines the side of a square $t_{1}[0,1]$

$t_{2} \quad$ value that determines the side of a square $t_{2}[0,1]$

$t_{i} \quad$ vertical component obtained from Hammersley strategy

$x\left(P_{i}\right) \quad$ function that determines the number of points enclosed in a $t_{1} \times t_{2}$ area

$x_{0}, y_{0}, z_{0} \quad$ Cartesian coordinates of the origin offsets

$x_{i}, y_{i}, z_{i} \quad$ Cartesian coordinates of the $i$ th measured point

$x_{p}, y_{p}, z_{p} \quad$ Cartesian coordinates of the $i$ th positioning point

$x y_{p} \quad$ horizontal component of the positioning coordinates 